\title{
Achievable secrecy rate of bit-interleaved coded modulation schemes
}

\author{
Weichen XIANG* , Stéphane Le Goff, Zhiguo DING \\ School of Electrical and Electronic Engineering, Newcastle University, NE1 7RU, UK
}

\begin{abstract}
We study the impact of various modulation mapping strategies and signal constellation shapes on the secrecy rates achievable with bit-interleaved coded modulation (BICM) schemes. Transmission over an ergodic Rayleigh fading channel is assumed throughout this work. Various constellations and mapping techniques are considered in this work to maximize the capacity difference between the main channel and the eavesdropper channel, rather than to optimize the capacity of both channels. We show that in terms of achievable secrecy rate, Gray and Quasi-Gray mappings only perform well at low SNR but outperformed by other mapping techniques when SNR increases. The proper design of signal mapping can significantly enhance the achievable secrecy rate in BICM schemes. It is indicated that the key parameter to the secrecy rate of a BICM system is the distance spectrum of Euclidean distances for mappings.
\end{abstract}

Key words: secrecy capacity; mapping; ergodic fading channel; bit-interleaved coded modulation

(C) 2012 JMT. All rights reserved.

\section{Introduction}

I n 1975, Wyner introduced the wiretap channel model, in which there are three users: the sender Alice, the legitimate receiver $B o b$, and the eavesdropper $E v e$ [1]. In this work, Wyner showed that if the channel between $\mathrm{Al}$ ice and Eve (eavesdropper channel) is a degraded version of the channel between Alice and Bob (main channel), it is possible to transmit information at a certain rate greater than zero in the main channel and yet achieve perfect secrecy. Lueng and Hellman extended Wyner's work in 1978 by considering the case where both main and eavesdropper channel are Gaussian. They were able to show that a positive secrecy rate can be achieved provided that the main channel is less noisy than the eavesdropper channel [2]. To obtain this result, Lueng and Hellman assumed the use of infinite-alphabet inputs.

The secrecy rate achievable with coded modulation (CM) schemes has been recently investigated under the assumption of finite-alphabet inputs, i.e. finite-size signal constellations [3]. Substantial differences have been found between the secrecy rate curves obtained with the schemes relying on finite-alphabet inputs and infinitealphabet inputs. It is well known that the information rate of a coded modulation system designed using an $M$ -

Received Oct. 19, 2012; revision accepted Nov. 4, 2012

${ }^{*}$ Corresponding author. E-mail: sunnyxwc@gmail.com (W.C. XIANG)

(C) 2012 JMT. All rights reserved doi: 10.3969/j.issn.2095-087X.2012.04.008 ary signal constellation cannot be higher than $\log _{2} M$ bits/channel use. This explains why the achievable secrecy rate of such system reduces to zero as the signal-tonoise ratio (SNR) of the main channel, denoted as $\gamma_{b}$ increases. In fact, increasing $\gamma_{b}$ also raises the eavesdropper channel $\gamma_{e}$, simply because the term $\gamma_{b}-\gamma_{e}(>0)$ is assumed to be constant. At SNR values that are sufficiently high, both main and eavesdropper channels have an achievable rate very close to $\log _{2} M$ bits/channel use, and the secrecy rate therefore drops to zero.

In this paper, we investigate the secrecy rate achievable with bit-interleaved coded modulation (BICM) schemes. Recall that BICM is a low complexity alternative to $\mathrm{CM}$ for bandwidth-efficient communications over fading channels [4]. In fact, it has been shown that, in most cases, the achievable information rates obtained with BICM are very close to those achieved with CM over both Gaussian and Rayleigh fading channels [5-6]. This is why the BICM approach has become today the de facto standard for designing wireless communication systems. Note that Gray mapping is the best mapping strategy for BICM systems as this is the labelling technique that optimizes the BICM information rates.

It is therefore interesting to investigate the issue of BICM design in the context of the wiretap channel where the sender Alice attempts to communicate with the legitimate receiver $B o b$, while hiding as much information as possible from the eavesdropper Eve. In particular, it is important to study the effects of both the 
signal constellation and the mapping technique on the secrecy rate. For instance, it already appears that Gray mapping may not be the best labelling strategy for the wiretap channel as it also maximizes the achievable rate for the eavesdropper channel. Other mapping techniques could offer a better performance than Gray labelling.

\section{System model}

An independent uniformly distributed (i.u.d.) binary source $S\left(P\left(S_{k}=1\right)=P\left(S_{k}=0\right)=0.5\right)$ generates an $N$ bit message sequence $M$ which is forwarded to the channel encoder. The corresponding encoder output $C$ is interleaved in a random fashion and then divided into $m$ bit sub-sequences that constitute the successive inputs to the signal mapper. The transmitter Alice maps the $m$-bit sequences onto the corresponding complex symbols $x$ and sends them through the main channel to Bob. Eve observes the communications between Alice and Bob through an eavesdropper channel and has the full knowledge of the signal constellation and labelling technique employed by Alice and Bob.

Throughout this paper, both main and eavesdropper channels are assumed to be independent Rayleigh fading channels that are both ergodic and non-frequency selective. We also assume that the average SNR in the eavesdropper channel is smaller than that of the main channel. In other words, the main channel is less noisy than the eavesdropper channel. The secrecy capacity $\hat{C}_{s}$ is simply defined as the difference between the information rate of the main channel, $C_{b}$, and the information rate, $C_{e}$, of the eavesdropper channel [1]:

$$
\hat{C}_{s}=C_{b}-C_{e} .
$$

For instance, when infinite-alphabet inputs are considered, we have $C_{b}=\log _{2}\left(1+\bar{\gamma}_{b}\right)$ and $C_{e}=$ $\log _{2}\left(1+\bar{\gamma}_{e}\right)$, where $\bar{\gamma}_{b}$ and $\bar{\gamma}_{e}$ are the average SNR values in the main channel and the eavesdropper channel, respectively. However, in case of finite-alphabet inputs, such as for CM or BICM, the expressions of $C_{b}$ and $C_{e}$ are more complicated and need to be evaluated using numerical integration methods. The channel transition functions of both channels are given by

$$
\begin{aligned}
& y_{b}=h_{b} x+n_{b}, \\
& y_{e}=h_{e} x+n_{e},
\end{aligned}
$$

where $h_{b}$ and $n_{b}$ are the Rayleigh fading coefficient and Gaussian noise affecting the symbol $x$ transmitted over the main channel, whereas $h_{e}$ and $n_{e}$ are the fading coefficient and Gaussian noise affecting the estimate of symbol $x$ received through the eavesdropper channel. Since the fading channel is assumed to be ergodic, different values of fading coefficients $h_{b}$ and $h_{e}$ will be assigned to each symbol that transmitted by Alice. Both noise samples $n_{b}$ and $n_{e}$ are Gaussian with a mean equal to zero and a variance of $\sigma^{2}$. Finally, $y_{b}$ and $y_{e}$ denote the estimates of $x$ available at the outputs of the main channel and eavesdropper channel, respectively. The Rayleigh fading channel is characterized by a transition probability density function $p(y \mid x)$ given by

$$
p(y \mid x)=\frac{1}{\pi \delta^{2}} \exp \left(-\frac{d^{2}(y, h x)}{\delta^{2}}\right),
$$

where $d^{2}(\cdot, \cdot)$ denotes the squared Euclidean distance operator, and the main channel and eavesdropper channel are both satisfy (4).

The channel capacity of a BICM scheme is given by [5]

$$
\hat{C}=m-\sum_{i=1}^{m} E_{c, y}\left\{\log _{2} \frac{\sum_{s \in \chi} p(y \mid s)}{\sum_{s \in \chi_{i}^{c_{j}}} p(y \mid s)}\right\},
$$

Where $c$ denotes the $m$-bit input to the mapper $\chi$, and $s \in \chi_{i}^{c_{j}}$ designates the subset of all signals $s \in \chi$, whose labels are equal to $c_{j} \in\{0,1\}$ in position $i$. We see that the squared Euclidean distance $d^{2}$ is an important factor in the BICM capacity computation. Before processing any further, it is worth recalling the definition of the distance spectrum $N\left(d_{e}\right)$, which is computed by

$$
N\left(d_{e}\right)=\frac{1}{m 2^{m}} \sum_{s_{i}^{c_{j}}} N_{h}\left(d_{e}, s_{i}^{c_{j}}\right),
$$

where $N_{h}\left(d_{e}, s_{i}^{c_{j}}\right)$ is the sum of all Hamming distances between the symbols $s_{i}^{c_{j}}$ and the symbols at Euclidean distance $d_{e}$ from $s_{i}^{c_{j}}$. The parameter $d_{e}$ takes many values in a large size constellation. However, we are most interested with the symbols with minimum Euclidean distance and second minimum Euclidean distance to $s_{i}^{c_{j}}$, we denote above Euclidean distances as $d_{e}(1)$ and $d_{e}(2)$, respectively.

It is shown in Ref. [5-6] that, in order to maximize the BICM capacity, it is essential to minimize the values of $d_{e}(1)$, which is simply done by employing Gray mapping, or quasi-Gray mapping whenever the constellation shape makes Gray labelling impossible. However, this holds only for traditional BICM systems without secrecy constraints. When those constraints exist, e.g. in the case of 
the wiretap channel model considered in our work, Gray/quasi-Gray labelling not only maximizes $C_{b}$ but also $C_{e}$. It is clear that a mapping technique that maximizes the channel capacity in the main channel while minimizing the channel capacity in the eavesdropper channel would lead to optimal secrecy information rates.

By combining Eqs. (1), (4) and (5), the secrecy capacity $\widehat{C}_{s}$ of BICM can be written as

$$
\widehat{C}_{s}=\sum_{i=1}^{m} E_{c, y}\left\{\log _{2} \frac{\sum_{s \in \chi} p\left(y_{e} \mid s\right) \sum_{s \in \chi_{i}^{\tau_{j}}} p\left(y_{b} \mid s\right)}{\sum_{s \in \chi} p\left(y_{b} \mid s\right) \sum_{s \in \chi_{i}^{\tau_{j}}} p\left(y_{e} \mid s\right)}\right\} .
$$

We divide (7) into two terms as follows

$$
\widehat{C}_{s}=\sum_{i=1}^{m}\left[F(\gamma)+G\left(N\left(d_{e}\right)\right)\right],
$$

the average constellation energy is designed to be 1 , so the noise variance $\delta^{2}=1 / 2 \gamma$. Thus, the $F(\gamma)$ and $G\left(N\left(d_{e}\right)\right)$ are connected with the SNR and squared Euclidean distances between received signal $y$ and the constellation points $s$, which are shown below:

$$
F(\gamma)=E_{c, y}\left\{\log _{2} \frac{\sum_{s \in \chi} \exp \left(-2 \gamma_{e} d^{2}\left(y_{e}, h_{e} s\right)\right)}{\sum_{s \in \chi} \exp \left(-2 \gamma_{b} d^{2}\left(y_{b}, h_{b} s\right)\right)}\right\},
$$

and

$$
G\left(N\left(d_{e}\right)\right)=E_{c, y}\left\{\log _{2} \frac{\sum_{s \in \chi_{i}^{c_{j}}} \exp \left(-2 \gamma_{b} d^{2}\left(y_{b}, h_{b} s\right)\right)}{\sum_{s \in \chi_{i}^{c_{j}}} \exp \left(-2 \gamma_{e} d^{2}\left(y_{e}, h_{e} s\right)\right)}\right\},
$$

where $\gamma_{b}$ and $\gamma_{e}$ designate the instantaneous SNR in the main channel and eavesdropper channel, respectively. In ergodic fading channels, we have

$$
\begin{aligned}
& \gamma_{b}=\left|h_{b}\right|^{2} \bar{\gamma}_{b}, \\
& \gamma_{e}=\left|h_{e}\right|^{2} \bar{\gamma}_{e} .
\end{aligned}
$$

\subsection{High SNR region}

At high SNR, we can write $y_{b} \approx h_{b} x$ and $y_{e} \approx h_{e} x$. In this case, Eq. (6) becomes

$$
\widehat{C}_{s} \approx \sum_{i=1}^{m} E_{c, y}\left\{\log _{2} \frac{p\left(y_{b} \mid x\right) p\left(y_{e} \mid x\right)}{p\left(y_{e} \mid x\right) p\left(y_{b} \mid x\right)}\right\}=0 .
$$

This result was expected, as for a finite size constellation, the capacities of both channels will ultimately reach $m$-bits/channel use at high SNR region.

\subsection{Medium SNR region}

Since $F(\gamma)$ considers all points on the constellation, its value is only affected by $\gamma_{b}$ and $\gamma_{e}$. However, the computation of $G\left(N\left(d_{e}\right)\right)$ only take into account of the signal points whose labels are equal to that of the transmitted symbol $x$ in position $i$. This indicates that the mapping technique does affect the achievable secrecy rate of a BICM scheme.

If we consider a particular position $i=1,2, \cdots, m$ in the label of signal points, the transmitted symbol $x$ has $l$ nearest neighbours denoted as $s_{n}^{k},(k=1,2, \cdots, l)$ that satisfy $s_{n}^{k} \in \chi_{i}^{c_{j}}$. We can now write $G\left(N\left(d_{e}\right)\right)$ as follows:

$$
\begin{gathered}
G\left(N\left(d_{e}\right)\right) \approx E_{c, y}\left\{\log _{2} \frac{p\left(y_{b} \mid x\right)+\sum_{k=1}^{l} p\left(y_{b} \mid s_{n}^{k}\right)}{p\left(y_{e} \mid x\right)+\sum_{k=1}^{l} p\left(y_{e} \mid s_{n}^{k}\right)}\right\} \\
\leq E_{c, y}\left\{\log _{2}\left(\frac{p\left(y_{b} \mid x\right)}{p\left(y_{e} \mid x\right)}+\max _{k=1}^{l} \frac{p\left(y_{b} \mid s_{n}^{k}\right)}{p\left(y_{e} \mid s_{n}^{k}\right)}\right)\right\} .
\end{gathered}
$$

Eq. (12) indicates that the effect of nearest symbols $s_{n}^{k} \in \chi_{i}^{c_{j}}$ is to reduce secrecy rate whenever

$$
p\left(y_{b} \mid s_{n}^{k}\right) \leq p\left(y_{e} \mid s_{n}^{k}\right) .
$$

It also shows that the maximum secrecy rate is achieved by minimizing the number of $s_{n}^{k}$, which satisfy the condition of $s_{n}^{k} \in \chi_{i}^{c_{j}}$. This strongly indicates that a mapping scheme that maximises the value of $N\left(d_{e}(1)\right)$ would lead to the highest achievable secrecy rate.

Simulation results are then provided by initially considering 16QAM signal set followed by the results on optimum mapping. For each 16-ary constellation, we also consider various well-known mapping techniques, namely Gray, set partitioning (SP) [7], modified setpartitioning (MSP) [8], maximum squared Euclidean weight (MSEW) [9] and M16 ${ }^{r}$ [10-11]. After that, we also provide some simulation results on 64QAM and show that finite-alphabet input with BICM scheme can achieve higher secrecy rate than infinite-alphabet input with Gaussian code.

\section{Numerical results}

In this section, we simulate the BICM secrecy rates for various signal constellations and mapping schemes over ergodic Rayleigh fading channels. Perfect channel side information (CSI) is assumed at both receivers and $\bar{\gamma}_{b}-\bar{\gamma}_{e}=5 \mathrm{~dB}$. 


\subsection{Example 1: 16QAM signal set}

We consider first the use of a 16QAM signal constellation. Gray, SP, MSP, MSEW and $M 16^{r}$ mappings can be employed in conjunction with this signal set.

The simulation results are showed in Fig. 1. These results indicate that, when $\bar{\gamma}_{b}$ is lower than $9 \mathrm{~dB}$, Gray mapping provides the highest secrecy rate among all mapping schemes considered here. However, as the $\bar{\gamma}_{b}$ is increased, it can be seen that the MSP, MSEW and $M 16^{r}$ mappings outperform Gray mapping at $9 \mathrm{~dB}$ while the SP mapping surpasses Gray mapping at $10 \mathrm{~dB}$. At $\bar{\gamma}_{b}=10 \mathrm{~dB}$, the maximum value of the secrecy rate with a Gray mapping is reached, while the secrecy rate values obtained with the other four mapping schemes still increase until a $\bar{\gamma}_{b}$ value of approximately $13 \mathrm{~dB}$. It is clear that $M 16^{r}$ mapping achieves the maximum secrecy rate value among all mapping schemes at $13 \mathrm{~dB}$, while Gray map-ping performs worst. $M 16^{r}$ mapping achieves higher secrecy rate than Gray mapping by approximately $0.2 \mathrm{bit} / \mathrm{channel}$ use. Eventually, as $\bar{\gamma}_{b}$ is further increased, all secrecy rates are progressively reduced to zero due to the finite size of 16QAM constellation.

A further comparison of the mapping schemes is on the first two terms of distance spectrum, which is defined in (5) (see Table 1). Gray mapping has minimum values of $N\left(d_{e}(1)\right)$ and $N\left(d_{e}(2)\right)$, while the parameters

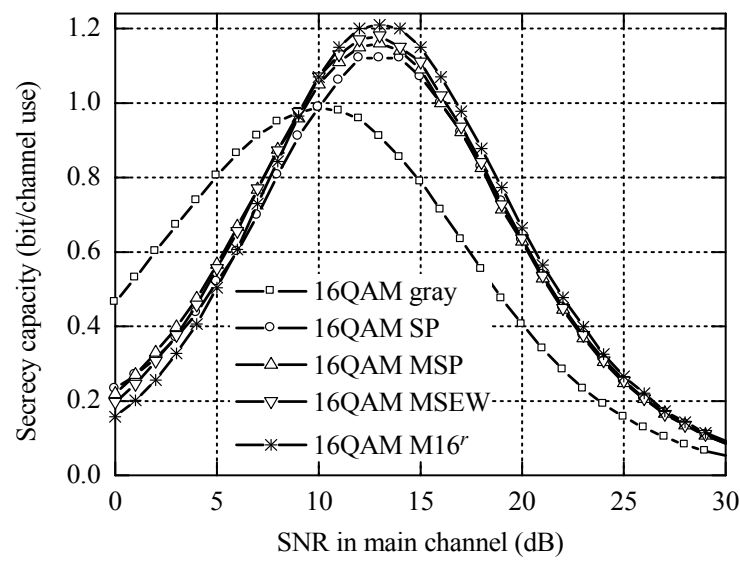

Fig. 1 Secrecy rate of various mapping schemes with 16QAM over ergodic Rayleigh fading channels

Table 1 Values of $N\left(d_{e}(n)\right)$ for some mappings with 16QAM

\begin{tabular}{cccccc}
\hline 16QAM & Gray & SP & MSP & MSEW & $M 16^{r}$ \\
\hline$N\left(d_{e}(1)\right)$ & 0.75 & 1.75 & 1.63 & 1.63 & 1.75 \\
$N\left(d_{e}(2)\right)$ & 1.88 & 2.75 & 2.81 & 2.94 & 3.06 \\
\hline
\end{tabular}

schemes, $M 16^{r}$ mapping greatly outperform Gray mapping at high SNR. MSP and MSEW mappings have same value of $N\left(d_{e}(1)\right)$ and very similar value of $N\left(d_{e}(2)\right)$, and they show same performances in Fig. 1. Then, we compare SP mapping and $M 16^{r}$ mapping, both of which have same value of $N\left(d_{e}(1)\right)$, but the value of $N\left(d_{e}(2)\right)$ of $M 16^{r}$ mapping is larger than that of SP mapping. The simulation results show that $M 16^{r}$ mapping gives higher achievable secrecy rate than SP mapping. Finally, For MSEW mapping and $M 16^{r}$ mapping, the values of $N\left(d_{e}(2)\right)$ for both of them are similar, but $M 16^{r}$ mapping has a larger value of $N\left(d_{e}(1)\right)$, and the achievable secrecy rate of $M 16^{r}$ mapping is higher.

We shall see that at medium to high SNR, $M 16^{r}$ continuously outperforms other mapping schemes, and more simulation results on other constellations are also provided.

\subsection{Example 2: optimum signal set}

Optimum constellation is constructed by distribute signal points in several hexagons, with points missing in some of them. On this constellation, Gray mapping is impossible (some constellation points have 6 nearest neighbours); thus, quasi-Gray is implemented instead. For other mapping schemes, if perfect mapping cannot be implemented, quasi mapping is used. The secrecy rate simulation results are shown in Fig. 2.

Quasi-Gray mapping shows good performance at low SNR, but the achievable secrecy rate is the lowest among all the mapping schemes. $M 16^{r}$ mapping achieves maximum value of secrecy rate of all mappings when $\bar{\gamma}_{b}=9 \mathrm{~dB}$. As usual, the value of $N\left(d_{e}(1)\right)$ and $N\left(d_{e}(2)\right)$ are computed and compared with the performances in Table 2, the $q$ - in it stands for quasi-.

Quasi-Gray mapping obtains the minimum values of $N\left(d_{e}(1)\right)$ and $N\left(d_{e}(2)\right)$ on optimum constellation, referring to the observation in 16QAM, and is expected to show the least value of achievable secrecy rate, as Fig. 2 confirms. $M 16^{r}$ mapping has the maximum value on both parameters among all mapping schemes and it gives the maximum value of achievable secrecy rate at $\bar{\gamma}_{b}=13 \mathrm{~dB}$.

The achievable secrecy rate of $M 16^{r}$ mapping is approximately 0.2 bit/channel use higher than Gray mapping. If we compare the performances in Figs. 1 and 2, the performances of $M 16^{r}$ mapping stay unchanged; however, the performance gap between Quasi-MSP and 
Quasi- $M 16^{r}$ becomes smaller. Quasi-MSP and quasiMSEW mapping have similar values on both parameters; they perform very close to each other. The $N\left(d_{e}(1)\right)$ value of quasi-Gray mapping is larger than quasi-Gray mapping but smaller than others, and it has the smallest value of $N\left(d_{e}(2)\right)$, which makes the achievable secrecy rate quasi-SP mapping outperforms quasi-Gray mapping, but much lower than other mapping schemes.

\subsection{Example 3: popular constellations vs. Gaussian input}

The examples on 16-ary signal sets have confirmed the result in (12), and we extend the examples to higher order modulation schemes. The high achievable secrecy rate is proposed to be obtained by employing D5 mapping [8] on 64QAM and $M 32^{r}$ mapping on 32QAM, and Gray mapping for each constellation is used for reference. In D5 mapping, the corresponding bit sequence of each symbol has a Hamming distance of 5, to any of its nearest neighbours. The $M 32^{r}$ mapping is generated by the same Binary Switch Algorithm (BSA) [7] as $M 16^{r}$ mapping. In this simulation, we also plot the secrecy capacity of using infinite-alphabet input. The results suggest that the secrecy rate achievable in BICM schemes is higher than with Gaussian input assumption.

With large values of $N\left(d_{e}(1)\right)=2.92$ and $N\left(d_{e}(2)\right)=$

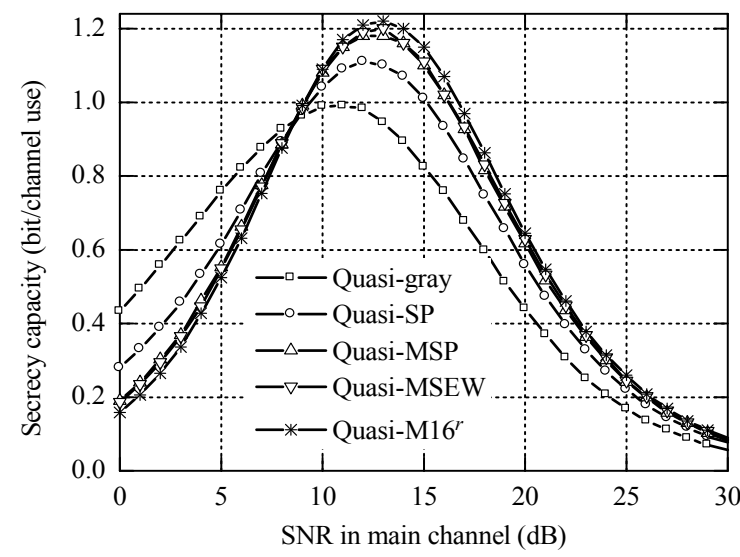

Fig. 2 Secrecy rate of various mapping schemes with optimum signal set over Ergodic Rayleigh fading channels

Table 2 Values of $N\left(d_{e}(n)\right)$ for some mappings with optimum

\begin{tabular}{cccccc}
\hline Optimum & Gray & SP & MSP & MSEW & $M 16^{r}$ \\
\hline$N\left(d_{e}(1)\right)$ & 1.41 & 2 & 2.28 & 2.31 & 2.43 \\
$N\left(d_{e}(2)\right)$ & 1.66 & 1.66 & 1.66 & 1.72 & 1.72 \\
\hline
\end{tabular}

3.94, D5 mapping obtains very high secrecy rate value at medium $\gamma_{b}$ and greatly outperforms other mapping schemes on achievable secrecy rate, it is even higher than the secrecy rate obtained by infinite size constellation. On the other hand, D5 mapping obtains very small secrecy rate value when $\bar{\gamma}_{b} \leq 5 \mathrm{~dB}$. The peak of the secrecy rate curve of D5 mapping is achieved at a $\bar{\gamma}_{b}=20 \mathrm{~dB}$, which is $5 \mathrm{~dB}$ larger than Gray mapping. Gray mapping has the smallest values of both $N\left(d_{e}(1)\right)=0.50$ and $N\left(d_{e}(2)\right)=1.60$, and achieves lower secrecy rate than that at high SNR. We now look at 32QAM: $M 32^{r}$ mapping outperforms Gray mapping on 32QAM at high SNR region. Table 3 shows that both values of $N\left(d_{e}(1)\right)$ and $N\left(d_{e}(2)\right)$ of $M 32^{r}$ are larger than Gray mapping. In Fig. 3, we see that the secrecy capacity obtained by Gaussian inputs is actually lower than the achievable secrecy rate of BICM schemes, this is not because BICM scheme breaks the channel capacity limits, but the various mappings on BICM scheme provide a practical way to limit the amount of information observed by eavesdropper, thus increasing the capacity difference between the main channel and eavesdropper channel.



Fig. 3 Secrecy rate of various mapping schemes with 32QAM and 64QAM signal sets over ergodic Rayleigh fading channels with the reference of Gaussian input. The SNR gap is $5 \mathrm{~dB}$.

Table 3 Values of $N\left(d_{e}(n)\right)$ for some mappings with 64QAM signal set

\begin{tabular}{cccccc}
\hline \multirow{2}{*}{$N\left(d_{e}(n)\right)$} & \multicolumn{2}{c}{ 64QAM } & & \multicolumn{2}{c}{ 32QAM } \\
\cline { 2 - 3 } \cline { 5 - 6 } & 64QAM & 32QAM & & 64QAM & 32QAM \\
\hline$N\left(d_{e}(1)\right)$ & 0.59 & 2.92 & & 0.75 & 1.70 \\
$N\left(d_{e}(2)\right)$ & 1.60 & 3.94 & 1.90 & 3.23 \\
\hline
\end{tabular}




\section{Conclusions}

The achievable secrecy rate of a BICM scheme obtained by Gray mapping is lower than other mappings. With constant SNR gap and perfect CSI at both receivers, mapping schemes with large values of $N\left(d_{e}(n)\right)$, especially when $n=1,2$, can obtain high secrecy rate performances on BICM system; these mapping schemes maximize the capacity differences between two channels, rather than optimize both channel capacities.

\section{References}

[1] A. Wyner, The Wire-tap channel, Bell System Technical Journal, 1975, 54(8): 1355-1387.

[2] S.K. Lueng-Yan-Cheong, M.E. Hellman, The Gaussian Wire-tap Channel, IEEE Trans. Inf. Theory, 1978, IT24(4): 451-456.

[3] S. Bashar, Z. Ding, C.S. Xiao, On secrecy rate analysis of MIMO wire-tap channels driven by finite-alphabet inputs, IEEE Trans. Commun., 2012, 99(1): 1-10.

[4] E. Zehavi, 8-PSK trellis codes for Rayleigh channel, IEEE Trans. Commun., 1992, 40(5): 873-884.
[5] G. Caire, G. Taricco, E. Biglieri, Bit-interleaved coded modulation, IEEE Trans. Commun., 1998, 40(3): 927-946.

[6] S.Y. Le Goff, Signaling constellation for powerefficient bit-interleaved coded modulations schemes, IEEE Trans. Inf. Theory, 2003, 49(1): 307-313.

[7] X. Li, A. Chindapol, J. Ritcey, Bit-interleaved coded modulation with iteration decoding, IEEE Commun. Lett., 1997, 1(6): 169-171.

[8] A. Chindapol, J.A. Ritcey, Design, analysis, and performance evaluation for BICM-ID with square QAM constellations in Rayleigh fading channels, IEEE J. Select. Areas in Commun., 2001, 19(5): 944-957.

[9] J. Tan, G.L. Stüber, Analysis and design of symbol mappers for iteratively decoded BICM, IEEE Trans. Wireless Commun., 2005, 4(2): 662-672.

[10] F. Schreckenbach, G. Bauch, Bit-interleaved coded irregular modulation, European Transactions on Telecommunications, 2006, 17(2): 269-282.

[11] F. Schreckenbach, N. Görtz, J. Hagenauer, et al., Optimized symbol mappings for bit-interleaved coded modulation with iterative decoding, In: IEEE Globecom Conference, San Francisco, USA, 2003: 3316-3320.

[12] S. Pfletschinger, F. Sanzi, Editorial: Error Floor Removal for Bit-Interleaved Coded Modulation with Iterative Detection, In: Proc. IEEE Trans. Wireless Commun., 2006, 5(11): 3174-3181.

(Editor: Yao ZHOU) 